Ann. Génét. Sél. anim., I972, 4 (4), 523-535.

\title{
LES MUTANTS GÉNIQUES A EFFETS VISIBLES DANS LES RACES BOVINES DE SUISSE
}

\author{
J.-J. LAUVERGNE et H. U. WINZENRIED \\ Laboratoire de Génétique factorielle, \\ Centre national de Recherches zootechniques, I. N. R. A., \\ 78350 Jouy en Josas, France \\ Institut de Zootechnie, \\ Université de Zurich \\ Winterthurerstr. 260, 8057 Zurich, Suisse
}

RÉSUMÉ

Les travaux génétiques sur les mutants à effet visible chez les bovins ont commencé en Suisse dans les années cinquante. Au début la plupart ont été réalisés à l'Institut de Zootechnie de la Faculté vétérinaire de Berne. Depuis 1965 le relais a été pris par l'institut homologue de la Faculté vétérinaire de Zurich.

C'est dans la race brune des Alpes (47 p. Ioo du cheptel suisse) que les travaux ont été le plus avancés : sur une hypoplasie de la mamelle, une affection oculaire, un albinisme. Une anomalie des spermatozoïdes et des variations colorés (ceinture et flancs colorés) sont également à l'étude.

En race Simmental tachetée rouge (49 p. Ioo du cheptel suisse), une hypoplasie des testicules a été récemment analysée et des travaux vont bientôt paraître sur un cas d'albinisme, définitivement non allèle de l'albinisme de la brune des Alpes. Un certain nombre de variants restent encore à étudier dans cette race, en particulier la frisure du poil et la brachygnathie.

Dans la race pie noire fribourgeoise (I,7 p. roo du cheptel suisse) d'effectif restreint, on a repéré, il y a quelques années déjà, des cas de dyschondroplasie héréditaire (veaux traîneaux) rapportables à un gène dont la fréquence était assez élevée, causant quelques dégâts.

La plupart de ces mutants ont disparu très rapidement, comme le veut la théorie lorsque les porteurs ont des coefficients de sélection très faibles. Ceux qui se maintiennnent après une durée assez longue, comme l'albinisme, la ceinture blanche et les flancs colorés en race brune des Alpes, semblent le faire par suite de l'établissement d'un équilibre ségrégationnel (l'hétérozygote étant avantagé naturellement ou artificiellement par rapport aux deux homozygotes). Dans le cas de la dyschondroplasie en race fribourgeoise, on invoquerait plutôt un effet de dérivé génétique dans une population d'effectif limité.

De nombreux points restent encore à élucider : le déterminisme même de certains caractères ainsi que les paramètres des lois de génétique des populations que suivent certaines fréquences géniques. 


\section{INTRODUCTION}

En dehors des polymorphismes biochimiques et sérologiques les travaux mendéliens sur les bovins ont, jusqu'a présent, principalement porté sur les anomalies héréditaires. On en compte plus de $25^{\circ}$ dans toute l'espèce (LAUVERGNE, I968).

En fait il est parfois difficile, à la limite, de faire la différence entre une anomalie et un caractère utile : par exemple l'hypertrophie musculaire sévèrement éliminée dans certaines races est, au contraire, recherchée dans d'autres. Il existe en outre des gènes déterminant les " traits " (cornage, couleur...) du standard des races sans influence sur la productivité en race pure puisqu'ils sont fixés mais qui vont ségréguer dans les croisements interraciaux de plus en plus à la mode.

Ainsi semble-t-il actuellement logique d'étudier plus généralement chez les bovins les mutants à " effet visible». Dans le présent article nous allons nous efforcer de passer en revue cette catégorie de gène dans les races bovines suisses.

\section{HISTORIQUE}

Comme c'est généralement le cas, les plus anciens travaux consacrés en Suisse à la tératologie bovine ont été faits dans les Écoles vétérinaires. Ils remontent au moins à I 900 (IAUVERGNE, I967) mais ne présentent guère d'intérêt pour notre propos avant la deuxième moitié du $\mathrm{xx}^{\mathrm{e}}$ siècle.

Le premier article où se manifeste une prise de conscience de l'existence de tels mutants en Suisse est dû en effet, en I946 à W. WEBER alors assistant à 1'Institut de Zootechnie de la Faculté vétérinaire de Berne. Devenu professeur et directeur de cet Institut cet auteur a, depuis, étudié un certain nombre de cas d'anomalies héréditaires en races brunes des Alpes (cécité), fribourgeoise (dyschondroplasie) et Simmental tachetée rouge (hypoplasie des testicules) tout en développant dans son Instien tut l'étude des polymorphismes décelables biochimiquement et sérologiquement et donnant également en son temps une courte revue des anomalies héréditaires suisses (WEBER, I963). Le premier en date des travaux consacrés à des cas indigènes reste cependant celui d'HÖFLIGER, professeur d'anatomie à la Faculté vétérinaire de Zurich, il a trait à une hypoplasie de la mamelle en race brune des Alpes. (HöFliGER, I952, a).

A 1'Institut de Zootechnie de la Faculté vétérinaire de Zurich (1) les premiers travaux consacrés aux gènes à effet visible remontent à r964 (à part une brève expérimentation avec un mâle brun des Alpes albinos). Cette année-là, date de la parution de l'article de WEBER et LAUVERGNE consacré à 1'albinisme en brune des Alpes de nouveaux cas de cette variation colorée firent leur apparition. On prit le parti de les recueillir afin de constituer un troupeau expérimental. Au fil des années ce troupeau s'est renouvelé et étoffé. Il a pu servir de base non seulement à l'étude de l'albinisme dans le bétail brun mais à des tests d'allélisme avec une autre forme d'albinisme apparu cette fois en Simmental.

(1) Il existe en Suisse 3 instituts d'enseignement supérieur et de recherche en zootechnie : dans chacune des deux Facultés vétérinaires de Berne et Zurich (Pr W. WeBER et Pr Winzenfied) et à l'École polytechnique fédérale de Zurich (Prs Lörtscher et F. WEBER). Ce dernier institut est le plus étoffé des trois, il préside à l'élaboration des plans de sélection des races domestiques à l'échelon national. 
L'étude d'autres mutants plus rares (alopécie en brune des Alpes, albinisme en Simmental déjà cité) a été également abordée, en même temps que 1'on s'orientait vers l'étude génétique de caractères de couleur à fréquence encore notable dans la race brune des Alpes : ceinture blanche et flancs colorés (à partir de r967).

Les travaux accomplis à 1'Institut de Zootechnie de la Faculté vétérinaire de Zurich sont le fruit d'une collaboration avec le Laboratoire de Génétique factorielle du Département de Génétique animale de 1'Institut national de la Recherche agronomique (I. N. R. A.) français. En outre, pour l'étude de l'albinisme une collaboration est amorcée avec 1'Institut de Zootechnie générale de la Faculté d'Agriculture de Milan (Pr SuccI). Des tests d'allelisme pour l'albinisme sont envisagés en liaison avec le Collège de Médecine vétérinaire de l'Université du Kansas (Prs HUSTON et LEIPOLD) organisme qui a la responsabilité d'un projet fédéral d'étude des anomalies bovines aux États-Unis.

\section{LES MUTANTS DÉCELÉS}

Deux principales races bovines se partagent le territoire helvétique, occupant en gros l'une la moitié ouest (la Simmental tachetée rouge) l'autre la moitié est du pays (la brune des Alpes). Elles totalisaient chacune entre 850000 et 900 ooo têtes en I966, soit, pour la tachetée rouge 49 p. Ioo des effectifs bovins et $47 \mathrm{p}$. Ioo pour la brune. La race fribourgeoise tachetée noire comptait, à cette même date, 30 ooo têtes (I,7 p. roo du cheptel total) (ANonyme, Ig68).

\section{I. - Mutants de la race Simmental tachetée rouge}

Ils sont présentés succinctement dans le tableau I.

TABLEAU I

Mutants à effets visibles en race Simmental, tachetée rouge, de Suisse

\begin{tabular}{|c|c|c|c|}
\hline Dénomination & $\begin{array}{l}\text { Déterminisme } \\
\text { héréditaire }\end{array}$ & Expansion dans la race & Références \\
\hline $\begin{array}{l}\text { Aplasie } \\
\text { de l'épididyme }\end{array}$ & $\begin{array}{l}\text { Autosomal } \\
\text { Récessif }\end{array}$ & $\begin{array}{l}18 \text { cas observés entre } 1957 \text { et } 1970 \\
\text { dont } 15 \text { bilatéraux }\end{array}$ & $\begin{array}{l}\text { KÖNIG }(1959,1961) \\
\text { KÖNIG et al. }(1972)\end{array}$ \\
\hline Brachygnathisme & Non établi & $\begin{array}{c}\text { Dans toute la race, fréquence phé- } \\
\text { notypique inférieure à } 0,1 \text { p. } 100\end{array}$ & $\begin{array}{l}\text { WEBER (1963) } \\
\text { GAILLARD (1972) }\end{array}$ \\
\hline Frisure du poil & Non établi & $\begin{array}{l}\text { Dans toute la race, fréquence géno- } \\
\text { typique de l'ordre de } 1 \text { p. } 100\end{array}$ & GAILLARD (1972) \\
\hline Nanisme & Non établi & A peu près disparu & $\begin{array}{l}\text { ANONYME }(1957) \\
\text { WEBER W. (1963) } \\
\text { WEBER F. (1972) }\end{array}$ \\
\hline Pseudo-albinisme & $\begin{array}{l}\text { Autosomal } \\
\text { Récessif }\end{array}$ & $\begin{array}{l}\text { Jusqu'à présent un seul cas apparu } \\
\text { spontanément }\end{array}$ & WEBER et al. (1973) \\
\hline
\end{tabular}


Jusqu'à présent, dans cette race un seul mutant a été étudié académiquement. Il s'agit (fig. I $a$ ) d'une aplasie des testicules, mutation récessive autosomale. La publication finale (KöNIG et al. I972) toute récente avait été précédée d'études partielles (KöNIG, I959, I960). Par ailleurs, un article sur une forme d'albinisme apparue pour la première fois en I969 dans le Canton de Berne (WEBER, I969) et qui a été évoqué plus haut devait bientôt voir le jour. Les accouplements expérimentaux mère $\times$ fils ont montré que ce trait était autosomal récessif. On a pu établir que ce mutant n'était pas allèle avec le mutant responsable de l'albinisme en race brune des Alpes également étudié à 1'Institut de Zootechnie de la Faculté vétérinaire de Zurich. D'ailleurs les deux types d'albinisme différent phénotypiquement déjà au niveau des yeux. Les yeux du mutants tacheté sont plus foncés que ceux du mutant brun avec hétérochromie de l'iris d'où le terme de pseudo-albinisme parfois employé pour le distinguer de l'autre type.

Deux mutants plus fréquents semble-t-il ségrègent encore dans la race : la frisure et la brachygnatie. Aucun d'entre eux n'a encore fait l'objet d'un début
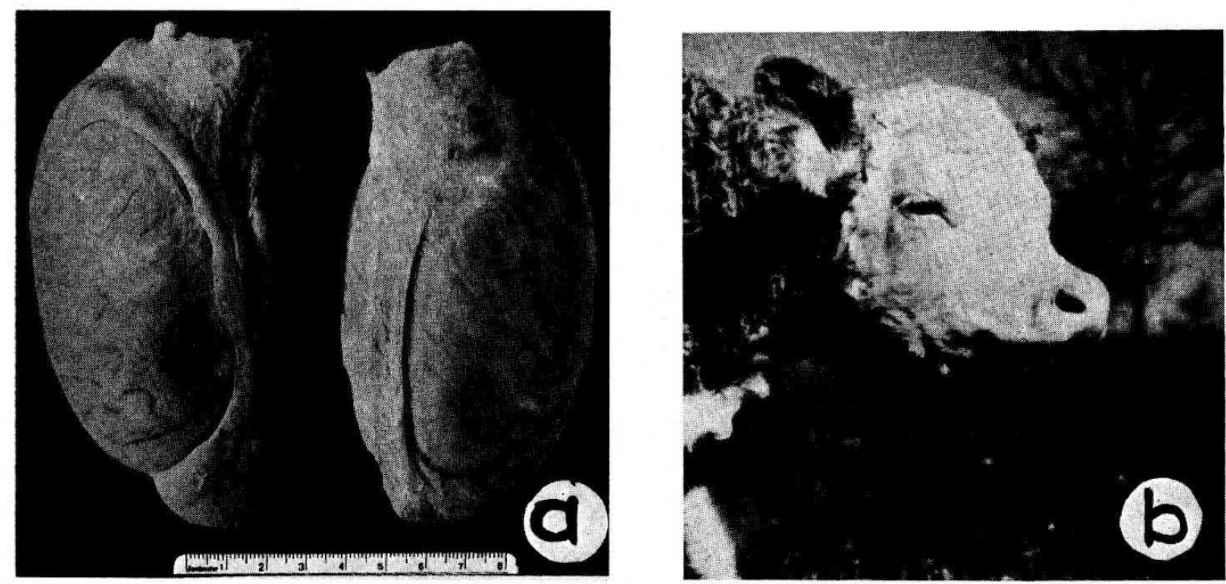

FIG. I. - a : aplasie testiculaire en race Simmental tachetèe rouge. Sur le testicüle droit dü taureau "BOBY " (2807 Rosière) on remarque l'aplasie de l'épididyme, le testicule gauche est normal. b : brachygnathie inférieure en race Simmental tachetée rouge. Clichés W. WEBER.
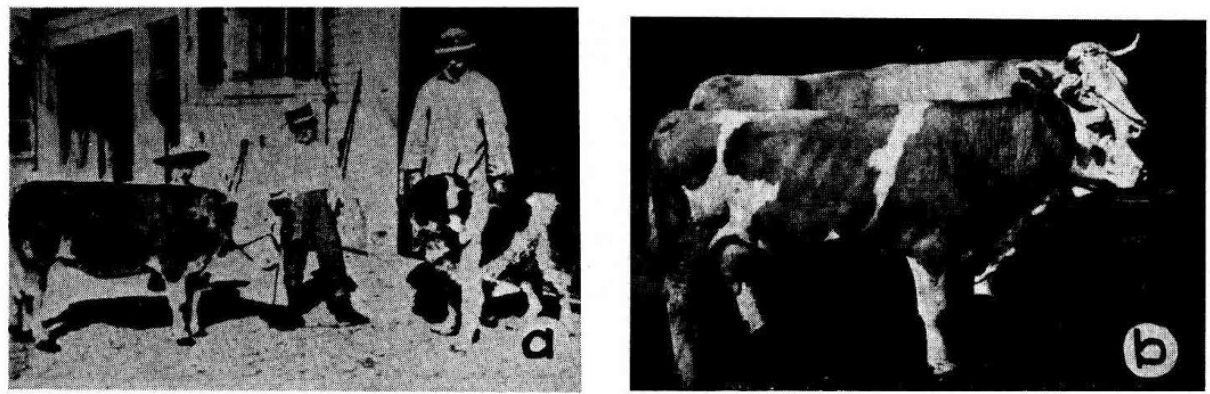

FrG. 2. - a : vue témoignant de l'ancienneté des apparitions de nains dans la race Simmental tachetée rouge. Comme il est impossible même de préciser si ce cas a une étiologie génétique on ne peut rien dire d'une éventuelle homologation avec les cas plus récents.

$\mathrm{b}:$ vache naine, Simmental tachetée rouge acquise en 1958 par la Station fédérale de la Chaman (Canton de Zoug) avec, en arrière plan, un témoin normal.

Clichés F. WEBER. 
d'étude génétique. La frisure est parfois assez populaire dans certains milieux d'éleveurs, encore qu'elle ne soit pas encouragée en insémination artificielle. Dans une statistique des causes d'élimination des taureaux d'IA portant sur 27 cas elle est évoquée cinq fois entre I962 et I966 (ANONymE, I966). La brachygnatie, déjà signalée par WEBER (I963), et considérée comme récessive par cet auteur est beaucoup plus rare (fig. I $b$ ).

Par le passé on a également connu des cas de nanisme (ANONYME, I957; WEBER, I963). Ils ne se rapportent pas forcément tous à une même origine génétique (cf. fig. 2 a). Un type au moins a fait l'objet d'une certaine sélection il y a une dizaine d'années, à l'instar de 1'attirance souvent manifestée par les éleveurs de tous les pays pour un bétail de type près du sol considéré à plus ou moins juste raison comme mieux "viandé ». Cette tentative a tourné court avant qu'on ait pu même préciser les caractéristiques exactes du mutant et son déterminisme héréditaire (fig. $2 b$ ).

\section{2. - Mutants de la race brune des Alpes}

C'est sur cette race qu'a, jusqu'à présent, porté l'essentiel des travaux de génétiques mendélienne. Les facteurs analysés sont listés dans le tableau 2.

Pour un défaut à expressivité variable - de 1'hypoplasie légère d'un seul quartier à l'aplasie totale de la mamelle (fig. 3) - limité au sexe femelle et sur lequel on ne dispose que de données recueillies dans les élevages il est bien difficile de conclure définitivement quant au déterminisme héréditaire. A juste titre, semble-t-il, KüNG (r956) avance 1'hypothèse de dominance autosomale à pénétrance incomplète qui semble meilleure que la récessivité simple précédemment invoquée.

Quant au leucome de la cornée, étudié par WEBER (Ig6o) sur 25 veaux, une transmission héréditaire monofactorielle autosomale dominante à pénétrance incomplète semble également jouer. Ce défaut a de nouveau, fait récemment sa réapparition (WINZENRIED, I972).

L'albinisme en race brune des Alpes (fig $3 a$ ) sur lequel les investigations remontent à I964 est actuellement le mutant le mieux étudié en Suisse où il a déjà fait 1'objet de 3 articles qui ne présentent encore qu'une faible partie des résultats maintenant acquis. Remontant dans le temps on a decélé plus de cinquante apparitions originales, la première datant de I929. Dans certains cas, avec 1'âge, se développe une certaine pigmentation dans le pelage. L'identité génétique d'une dizaine d'apparitions fortuites a été établie et l'on voit que le gène est répandu avec une égale densité dans tout le domaine de la race avec, dans certaines zones, des concentrations d'homozygotes peut-être en rapport avec des élévations locales du coefficient de consanguinité (Appenzell Rhodes intérieures et Grisons). Les inconvénients de l'albinisme pour les porteurs semblent minimes : on a relevé plusieurs cas de femelles ayant fait des carrières analogues à celles des reproductrices normales. Connaissant les pedigrees d'une trentaine d'albinos on s'efforce actuellement de déterminer l'ancêtre commun à l'aide d'un programme mécanographique qui nous permettra de remonter jusqu'à 20 générations. Bien que la fréquence génique ne soit pas entièrement négligeable (entre $0, \mathrm{I}$ et I p. IOO), comme le caractère est récessif, la fréquence génotypique est très faible. On s'explique encore mal les causes de son extension à travers toute la race. L'hypothèse de dérive génétique doit toutefois être écartée, semble-t-il. 
TABLEAU 2 Mutants à effets visibles en race brune des Alpes de Suisse

\begin{tabular}{|c|c|c|c|}
\hline Dénomination & $\begin{array}{l}\text { Déterminisme } \\
\text { héréditaire }\end{array}$ & Extension dans la race & Références \\
\hline Albinisme & $\begin{array}{l}\text { Autosomal } \\
\text { récessif }\end{array}$ & $\begin{array}{l}\text { Fréquence génique de } 0,1 \text { à } \\
1 \text { p. } 100 \\
\text { Fréquence de l'homozygote } \\
\text { albinos } 1 / 200000\end{array}$ & $\begin{array}{l}\text { WEBER et LAUVERGNE (1964) } \\
\text { ZANOTTI } \text { et al. (1967) } \\
\text { WINZENRIED et LAUVERGNE (1968) } \\
\text { BONADONNA et al. (1969) } \\
\text { ORIZOTTI } \text { et al. (1969) } \\
\text { WINZENRIED et LAUVERGNE (1970) } \\
\text { BonADONNA et SUCCI (9171 } \\
\text { (No } 22 \text { du catalogue de LAUVERGNE } \\
\text { (1968) } \\
\text { En cours d'étude à la fois à l'Ins- } \\
\text { titut de Zootechnie, Faculté vétéri- } \\
\text { naire de Zurich et à l'Institut de Zoo- } \\
\text { technie générale de Milan }\end{array}$ \\
\hline Alopécie & $\begin{array}{l}\text { Peut-être auto- } \\
\text { somal récessif }\end{array}$ & $\begin{array}{l}\text { Observé une seule fois jus- } \\
\text { qu'à présent }\end{array}$ & $\left\{\begin{array}{l}\text { Observation faite à l'Institut de Zoo- } \\
\text { technie, Faculté vétérinaire de Zurich }\end{array}\right.$ \\
\hline $\begin{array}{l}\text { Ceinture blanche } \\
\text { ("Gurt") }\end{array}$ & $\begin{array}{l}\text { Dominant autoso- } \\
\text { mal à expres- } \\
\text { sivité variable et } \\
\text { pénétrance sub- } \\
\text { complète }\end{array}$ & $\begin{array}{l}\text { Dans certaines populations } \\
\text { non inscrites de la Suisse } \\
\text { du Nord-Est, fréquence } \\
\text { génique de } 0,25 \text { p. } 100, \\
\text { très rare dans le cheptel } \\
\text { inscrit où il donne parfois } \\
\text { des taches blanches au } \\
\text { ventre }\end{array}$ & $\begin{array}{l}\text { LAUVERGNE et WINZENRIED (1969) } \\
\text { En cours d'étude à l'Institut de Zoo- } \\
\text { technie, Faculté vétérinaire, de Zu- } \\
\text { rich) }\end{array}$ \\
\hline Ectromélie & $\begin{array}{l}\text { Récessif } \\
\text { autosomal }\end{array}$ & $\begin{array}{l}\text { Trois cas observés en Tur- } \\
\text { quie en } 1960 \text { dans la des- } \\
\text { cendance de bétail im- } \\
\text { porté de suisse }\end{array}$ & $\begin{array}{l}\text { Düzgünes et Tuncay (1962) } \\
\text { (No } 82 \text { du catalogue de LAUvERGne } \\
\quad(\mathbf{1 9 6 8 )})\end{array}$ \\
\hline $\begin{array}{l}\text { Flancs colorés } \\
\text { (" Bluem ") }\end{array}$ & $\begin{array}{l}\text { Dominant } \\
\text { autosomal à } \\
\text { expressivité varia- } \\
\text { ble et pénétrance } \\
\text { complète ou sub- } \\
\text { complète }\end{array}$ & $\begin{array}{l}\text { Dans les mêmes popula- } \\
\text { tions que celles où l'on } \\
\text { trouve la ceinture, avec } \\
\text { la même fréquence }(0,25 \\
\text { p. } 100) \text {, ne semble pas } \\
\text { exister dans le cheptel } \\
\text { inscrit }\end{array}$ & $\begin{array}{l}\text { LAUVERGNE et WINZENRIED (1969) } \\
\text { En cours d'étude à l'Institut de } \\
\text { Zootechnie, Faculté vétérinaire de } \\
\text { Zurich) }\end{array}$ \\
\hline $\begin{array}{l}\text { Hypoplasie } \\
\text { de la mamelle }\end{array}$ & $\begin{array}{l}\text { Dominant autoso- } \\
\text { mal à pénétrance } \\
\text { incomplète et ex- } \\
\text { pressivité varia- } \\
\text { ble limitée au } \\
\text { sexe femelle }\end{array}$ & Probablement disparu & $\begin{array}{l}\text { HÖFLIGER }(1952 a, b) \\
\text { KüNG }(1956) \\
\text { (No } 133 \text { du catalogue de LAUVERGNE } \\
\quad(1968))\end{array}$ \\
\hline $\begin{array}{l}\text { Leucome cornéen } \\
\text { (cécité) }\end{array}$ & $\begin{array}{l}\text { Dominant autoso- } \\
\text { mal à expressi- } \\
\text { vité variable et } \\
\text { pénétrance in- } \\
\text { complète }\end{array}$ & Récemment réapparu & $\begin{array}{l}\text { WEBER }(1960) \\
\text { (No } 128 \text { du catalogue LAUVERGNE } \\
(1968) \text { ) } \\
\text { WINZENRIED }(1972)\end{array}$ \\
\hline Sans cornes & $\begin{array}{l}\text { Dominant } \\
\text { autosomal }\end{array}$ & $\mid \begin{array}{l}\text { Apparu en Afrique du Sud } \\
\text { dans la descendance de } \\
\text { bétail importé de Suisse. } \\
\text { Une sous-race présentant } \\
\text { le caractère est en voie de } \\
\text { constitution }\end{array}$ & ANONYME (1970) \\
\hline $\begin{array}{l}\text { Spermatozoïdes } \\
\text { à tête géante } \\
\text { et à double queue }\end{array}$ & $\begin{array}{l}\text { Dominant } \\
\text { autosomal }\end{array}$ & $\begin{array}{l}\text { Observé en } 1968 \text { chez un } \\
\text { mâle et, depuis, chez } 3 \text { de } \\
\text { ses descendants }\end{array}$ & $\begin{array}{l}\text { BERTSCHINGER (1972) } \\
\text { En cours d'étude à l'Institut de } \\
\text { Zootechnie de la Faculté vétérin zire } \\
\text { de Zurich }\end{array}$ \\
\hline
\end{tabular}


Ce gène a aussi été détecté en Italie du Nord dans la descendance d'animaux qui tirent leur origine de Suisse. Plusieurs articles lui ont d'ailleurs déjà été consacrés dans ce pays. Depuis I97I nous l'avons vu, les travaux italiens sont couplés avec les travaux suisses.
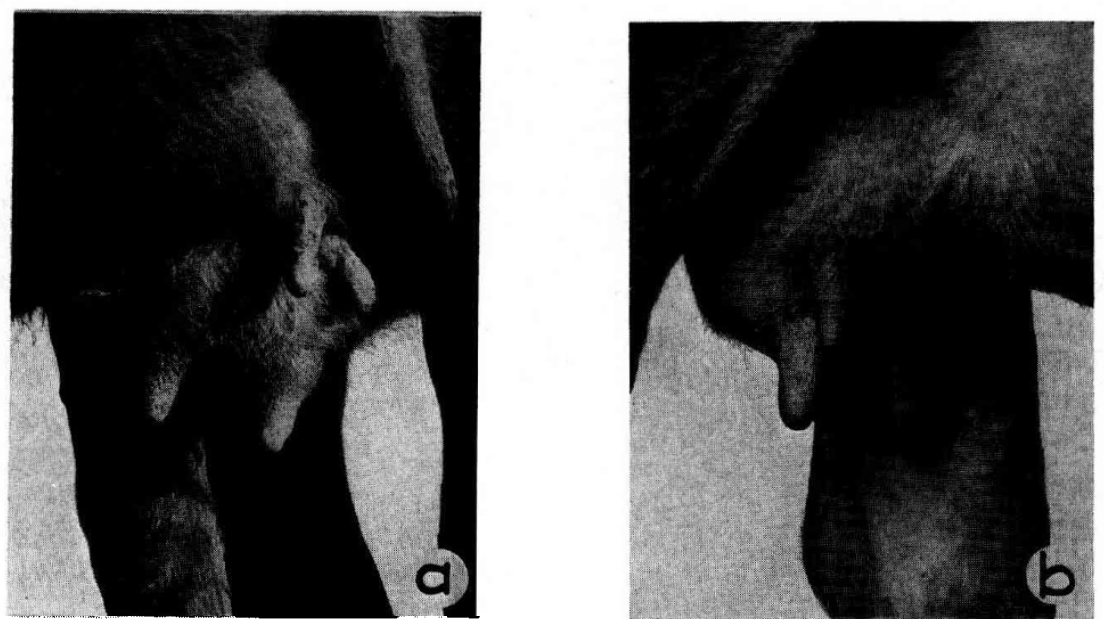

Fig. 3 a et b. - Deux cas d'hypoplasie héréditaire de la mamelle en race brune des Alpes Clichés H. HÖFliger

Rappelons que le gène d'albinisme en brune des Alpes n'est pas allèle du gène du pseudo-albinisme de la Simmental tachetée rouge.

Bien que la standard coloré soit brun uniforme et que, pour la monte publique, on n'emploie que des animaux de ce type, il est toute une région - où dominent les petits éleveurs non inscrits aux syndicats d'élevage — où 1'on trouve deux variations de panachure blanche typiques : la ceinture (appelée "Gurt ") et les flancs
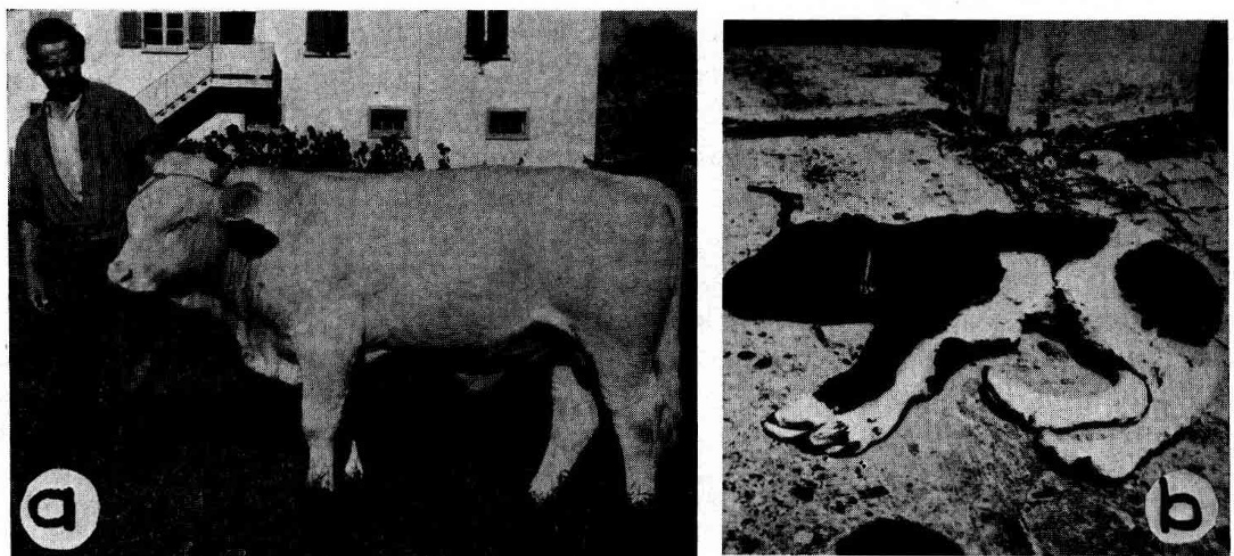

FIG. 4. - a : mâle albinos brun des Alpes utilisé dans les premières expériences de croisements en 1957 par le Pr WINZENRIED.

Cliché : H. U. WinzenRIED.

b : Veau traîneau (dyschondroplasie) en race fribourgeoise pie noire. Cliché F. Weber. 
colorés ("Bluem 》) (fig. 5). L'étude génétique entreprise en I967, forcément lente - car il s'agit d'enquête auprès d'éleveurs très dispersés qui ont chacun une ou deux vaches panachées -, montre que ces deux couleurs sont sous l'influence de gènes dominants dont l'expressivité est variable et, à la limite, la pénétrance subcomplète, au moins pour la ceinture. Dans le Toggenbourg (Canton de Saint-Gall) la fréquence de chacun de ces gènes est de 1'ordre de o,25 p. Ioo, ce qui donne environ 0,5 p. Ioo de Gurt et 0,5 p. Ioo de Bluem : au total I p. Ioo d'animaux panachés (LAUVERGNe et WINZENRIED (I969).
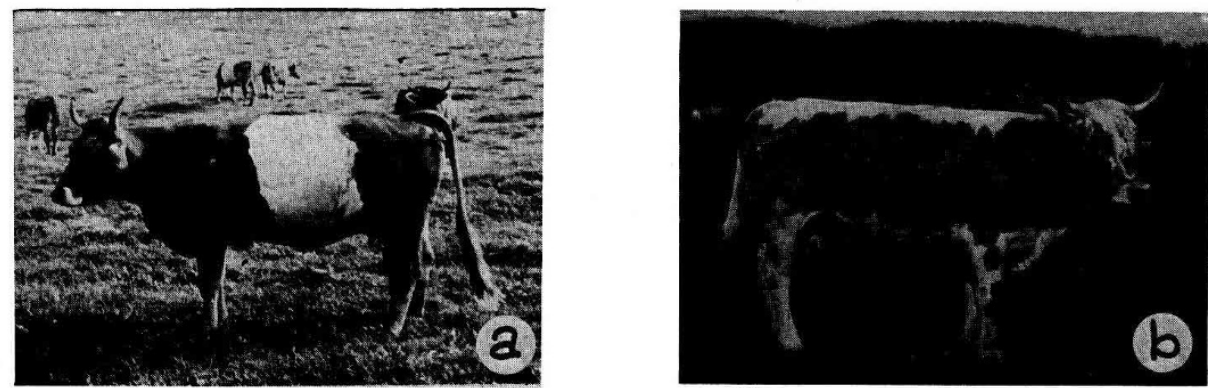

FIG. 5. - a : panachure "Gurt" ceinture bianche en race 'prune des Àlpes àe Ŝisse. b : panachure "Bluem": flancs colorés en race brune des Alpes de Suisse. Clichés : J.-J. LAuvergne.

L'étude du comportement du gène pour la ceinture blanche montre qu'il est sans doute responsable des taches blanches ventrales apparaissant parfois chez les animaux inscrits, par une action dominante à pénétrance incomplète et expressivité réduite. Ces taches ont, par le passé, donné quelque souci aux sélectionneurs du Herd-Book.

En I968 BERTSCHINGER dans le cadre des examens de routine de la semence à 1'Institut de Zootechnie de la Faculté vétérinaire de Zurich découvrait dans le sperme faiblement fécondant du taureau "ACHIM " 20 p. Ioo de spermatozoïdes à tête géante (fig. $6 a$ ) dont la moitié avec une double queue (fig. $6 b$ ). La fréquence de ces défauts restait constante dans la semence avec les années.

Le sperme de 6 fils d'“ ACHIM " a déjà pu être examiné. Chez trois d'entre eux 1'anomalie paternelle se retrouvait alors que les trois autres semblaient parfaitement normaux. L'hỵpothèse actuellement formulée par BERTSCHINGER (I972) qui poursuit les analyses en vue d'un travail de thèse est celle de monofactorialité autosomale en dominance.

A l'étranger jusqu'à présent deux mutations (1) ont été signalées, qui n'ont pas encore été décrites en Suisse. L'une est l'absence de corne dans le bétail brun importé en Afrique du Sud. La première apparition d'un animal motte remonterait à 1943 , le caractère serait monofactoriel autosomal dominant et une sous-race motte est en voie de création (ANONYME, r970). L'autre est 1'ectromélie, brièvement

(1) On ne s'occupe ici que des rameaux bruns des Alpes récemment détachés de la race et qui s'alimentent encore en reproducteurs en Suisse, laissant de côté en particulier le rameau brun des Alpes des États-Unis, isolé de ses origines depuis plus d'un siècle maintenant. Toutefois, si l'introduction en Suisse de ce type de bétail brown swiss déjà amorcée devait s'intensifier il serait bon que les praticiens suisses se tiennent au courant des mutants à effets visibles existant dans cette race. La même remarque est valable en race tachetée rouge pour laquelle la Suisse, longtemps seulement exportatrice, commence à échanger des reproducteurs avec d'autres pays. 
décrite en Turquie par DüzGÜNES et Tuncay (I962) et observée chez des veaux apparentés et assez consanguins. Ce défaut récessif autosomal ne semblait pas accompagné d'autres déformations osseuses, au contraire de l'ectromélie bien connue en Frisonne pie noire.
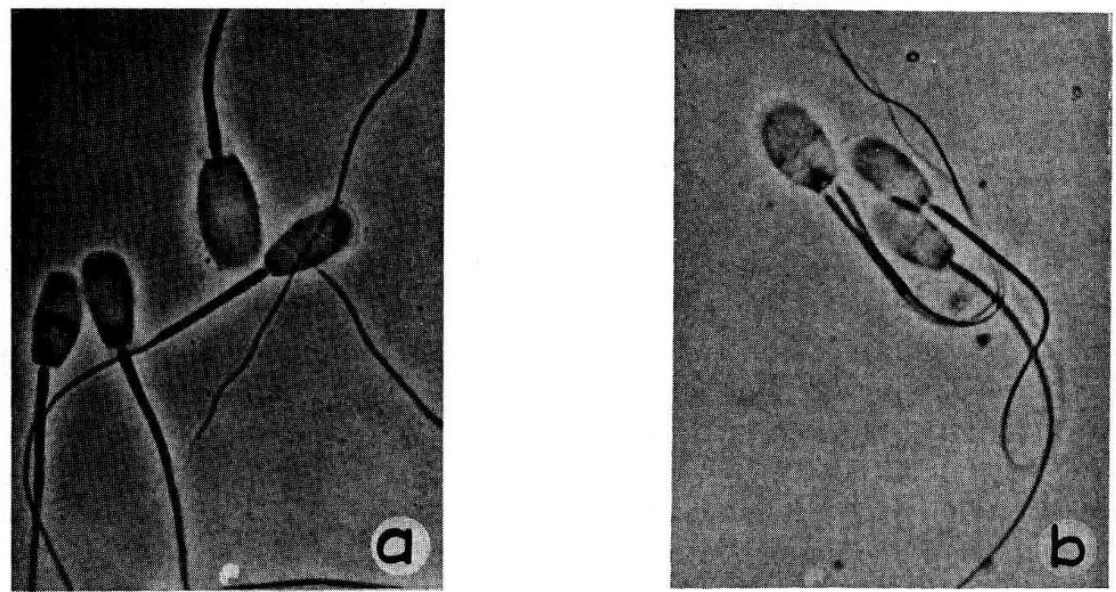

FIG. 6. - Tête géante et double queue čnez les spermatozö̈des du taureau brun des Alpes " $A C H I M$ " et de ses descendants. a. Spermatozoïde à tête géante

b. Spermatozoìde à double queue et tête géante

Clichés H. J. Bertschinger.

Pour en finir avec la race brune des Alpes il faut mentionner qu'en r970 un veau femelle sans poils a été recueilli par 1'Institut de Zootechnie de la Faculté vétérinaire de Zurich. Malheureusement, suite à des ennuis digestifs, 1'animal a dû être précocement abattu, ce qui n'a pas permis d'en étudier l'éventuel déterminisme génétique. L'expérience montrant, qu’à l'instar des albinismes, les alopécies congénitales ont fréquemment une étiologie mendélienne. il a semblé utile de mentionner cette apparition.

\section{3. - Race fribourgeoise pie noire}

L a seule anomalie connue dans cette race est récessive autosomale létale (fig. $3 b$ ). Souvent elle est fatale pour la mère lors du part.

TABLEAU 3

Mutants à effets visibles en race fribourgeoise tachetée noire de Suisse

\begin{tabular}{|c|c|c|c|}
\hline Dénomination & $\begin{array}{c}\text { Déterminisme } \\
\text { héréditaire }\end{array}$ & $\begin{array}{l}\text { Expansion } \\
\text { dans la race }\end{array}$ & Références \\
\hline $\begin{array}{l}\text { Dyschondroplasie } \\
\text { (veaux traîneaux) }\end{array}$ & $\begin{array}{l}\text { Autosomal } \\
\text { Récessif }\end{array}$ & $\begin{array}{r}\text { Plus ou moins } \\
\text { latent encore } \\
\text { dans la race. }\end{array}$ & $\begin{array}{l}\text { WEBER }(1962) \\
\text { WEBER 1963) } \\
\text { (No } 147 \text { du catalogue } \\
\text { LAUVERGNe (1968) }\end{array}$ \\
\hline
\end{tabular}




\section{4. - Autres études}

Notre revue resterait incomplète si nous ne mentionnions pas la contribution de WEBER (I963) à l'étude du mutant “bulldog » Dexter de la race Kerry qu'il eut l'occasion d'examiner dans un troupeau du Valais.

\section{DISCUSSION}

Les bovins domestiques ont un long intervalle de génération et une prolificité femelle réduite. A la fois reproductrices, productrices et éléments dans les plans de sélection les femelles se renouvellent lentement. Quant au choix des taureaux, qui se fait de plus en plus sur examen de la descendance, il ne permet pas, en général, de déterminer la formule génique des animaux.

Ainsi, dans cette espèce, la fréquence d'un gène nouvellement introduit pourrat-elle évoluer, au moins partiellement, selon des lois de génétique des populations naturelles voisines de celles qui prévalent chez 1'Homme, échappant au contrôle des éleveurs et des sélectionneurs. Si, dans ce schéma-là, la plupart des nouveaux mutants généralement défavorables sont appelés à disparaître rapidement la théorie et l'expérience montrent que d'autres peuvent, dans certaines conditions, atteindre des fréquences qui se fixent au moins temporairement à des valeurs d'équilibre non négligeables. Il y a risque de pertes économiques et de perturbations des plans de sélection.

La plupart des mutants détectés en Suisse se rangent dans la première catégorie : les gènes en voie de disparition. C'est sans doute le cas, par exemple, de l'hypoplasie de la mamelle, de 1'alopécie, de 1'anomalie du sperme décrite par BERTSCHINGER en race brune des Alpes, du pseudo-albinisme et de l'aplasie testiculaire en tachetée rouge. Dans la plupart de ces cas l'ancêtre commun est proche, ce qui indique que le mutant n'existe pas depuis très longtemps.

Par contre dans d'autres situations analogues à certaines de celles que LALOUEL (I97I) a analysées chez 1'Homme il semble bien y avoir au moins un début de fixation de la fréquence autour d'une certaine valeur d'équilibre.

Ainsi la dérive aléatoire qui a lieu dans les populations d'effectif limité pourrait expliquer la concentration de la dyschondroplasie (veaux traîneaux) en race Pie noive fribourgeoise.

Ainsi l'équilibre ségrégationnel pourrait expliquer les équilibres observés pour 1'albinisme, la ceinture blanche et les flancs colorés en race brune des Alpes ainsi que pour la frisure en tachetée rouge.

Dans le premier cas (l'albinisme) on doit exclure une action, même involontaire, de 1'Homme en faveur du mutant puisque la population inscrite dans laquelle il se maintient exclut de ses rangs tous les albinos. Il faut supposer que les hétérozygotes, vraisemblablement les femelles, possèdent un avantage (pour la reproduction et/ou la longévité, et/ou la production) sur les homozygotes normales.

Quant à l'équilibre ségrégationnel observé pour la ceinture, les flancs colorés ou la frisure il est dû principalement à la volonté des éleveurs, ce qui peut éventuellement masquer un désavantage sélectif naturel ou aller de pair avec un avantage. 
A l'issue de cette brève analyse on note que de nombreuses questions restent encore sans réponse. Il est toutefois intéressant de remarquer à propos de l'albinisme en brune des Alpes, dont l'étude est bien amorcée, que les analyses de génétique de population pourront aider à mettre en lumière la structure de parenté de la race.

Reçu pour publication en mai 1972.

\section{SUMMARY}

\section{GENIC MUTATIONS WITH VISIBLE EFFECT IN SWISS CATTLE BREEDS}

Genetic studies on mutations with visible effect in cattle were begun in Switzerland in the 1950's. In the beginning, most of them were done at the Animal Husbandry Institute of the Faculty of Veterinary Medicine at Berne. Since 1965 these studies have been continued by the similar institute in the Veterinary Medicine Faculty at Zurich.

In the Alpine Brown breed (47 p. Ioo of Swiss cattle), hypoplasia of the udder, eye disease and albinism have been most studied. A spermatozoid abnormality and color variations (belted and color-sided) are also under study.

In the Simmental red pied breed (40 p. 100 of Swiss livestock), hypoplasia of the testicles was analyzed recently, and studies will soon be published on a case of definite non-allele albinism as compared with Alpine Brown albinism. There are still a number of variations to study in this breed, in particular hair curliness and brachygnathia.

In the Fribourg black pied breed which are few in number (I.7 p. Ioo of Swiss cattle), cases of hereditary dyschondroplasia were found several years ago (sled calves) due to a gene having a rather high frequency, causing some damage.

Most of these mutations disappeared very quickly when the carriers had very low selection coefficients. Those like albinism, white belt, and color sided in the Alpine Brown breed which remain a long time, seem to be controlled by segregational balance, the heterozygote being naturally or artificially favored as compared to two homozygotes. Dyschondroplasia in the Fribourg breed might rather be due to genetic drift in a small population.

Many points still remain to be cleared up such as the determinism of some characters as well as the parameters of genetic population laws which follow certain genic frequencies.

\section{ZUSAMMENFASSUNG}

\section{SICHTBARE GENMUTATIONEN BEI DEN SCHWEIZERISCHEN RINDERRASSEN}

Die wissenschaftliche Erforschung und genetische Abklärung von sichtbaren Genmutationen bei den schweizerischen Rinderrassen hat ihren Anfang in den 5oer-Jahren genommen. $\mathrm{Zu}$ Beginn befasste sich vor allem das Tierzucht Institut der Veterinär-medizinischen Fakultät von Bern mit diesen Problemen, wurde jedoch seit 1965 vom Schwesterinstitut der Veterinärmedizinischen Fakultät von Zürich abgelöst.

Ein grosser Teil der Untersuchungen befasst sich mit Genmutationen beim schweizerischen Braunvieh (47 p. Ioo des Gesamtrinderbestandes der Schweiz), wo vor allem Fälle von Hypoplasien des Euters, Augenerkrankungen, Farbvarietfäten (Albinismus, Gurt und Bluem) und Sperma-Anomalien beschrieben wurden.

Beim Simmentaler Fleckvieh (49 p. Ioo des Gesamtrinderbestandes) sind Hypoplasien der Hoden untersucht worden, und bald werden Arbeiten über einen Fall von Albinismus, der kein Allel bildet zum Albinismus beim Braunvieh, erscheinen. Weitere Genmutationen sind gegenwärtig im Studium, so zum Beispiel die Rauhhaarigkeit und die Brachygnatie.

Beim Freiburger Schwarzfleckrind (I,7 p. Ioo des Gesamtrinderbestandes) wurden vor einigen Jahren Fälle von Dyschondroplasie, welche auf ein Gen mit einer ziemlich hohen Frequenz zurückzuführen sind, beschrieben. 
Die meisten sichtbaren Genmutationen verschwinden relativ rasch, weil sie in der Regel einen kleinen Selektionskoeffizienten aufweisen. Albinismus, Gurt und Bluem beim schweizerischen Braunvieh konnten sich wahrscheinlich deshalb bis heute halten, weil die Heterozygoten, sowohl in der natürlichen als auch in der künstlichen Selektion, gegenüber den Homozygoten bevorteilt wurden. Bei der Dyschondroplasie der Freiburger Rass ist man eher geneight, von Gen-Abgleitung in einer zahlenmässig limitierten Population zu sprechen.

Die Abklärung der genetischen Parameter und der Genfrequenzen der beobachteten Mutationen bedürfen noch einer näheren Untersuchung.

\section{REMERCIEMEN'TS}

Les professeurs W. WEBER (Institut de Zootechnie, Faculté vétérinaive de Berne) et F. WEBER (Institut de Zootechnie, Éçle polytechnique fédérale, Zurich) ont lu le manuscrit et suggéré de nécessaires retouches. Ils ont également fourni une partie de l'iconographie. On voudrait également remercier le professeur Hörliger (Institut d'Anatomie, Faculté vétérinaive de Zurich) et M. Cl. Gaillard, Fédération suisse pour l'I. A. Zollikofen (BE).

\section{RÉFÉRENCES BIBIOGRAPHIQUES}

Anonyme. I957. L'apparition du nanisme et sa lutte dans la race tachetée du Simental. Bull. Féd. Suisse élev. race tachetée rouge (5), 2425 (rapport du Dr. Lörtscher).

Anonyme, 1966. Causes d'élimination des taureaux d'IA (1. 1. 1962-30. 6. 1966). IA, Bulletin de la Fédération Suisse pour l'Insémination artificielle 4 (3), 24-26,

Anonyme, 1968. Le cheptel suisse. Bureau fédéral de Statistique, Berne. Statistiques de la Suisse, 42 I $^{\mathrm{e}}$ fasc., série Ch8.

Anonyme, r97o. Poenskop-Swisters. Produseer dat dit Klap. Landbou-weekblad, I40-I42.

Bertschinger H. J., I972. Communication personnelle.

Bonadonna T., Roy Choudhury P. N., Succi G., I969. Osservazioni su talune caratteristiche del materiale spermatico di un toro albino di razza bruna delle alpi. Boll. Soc. ital. Biol. Sperim., 46, 49-5I.

Bonadonna T., Succi G., I97i. Certaines caractéristiques somatiques et comportement sexuel des bovins albinos. $X^{\mathrm{e}}$ Congr. int. Zootech. Versailles, Communications libres, thème VII.

Düzgünes O., TuncaY A., I962. The amputated condition in brown swiss cattle. J. Hered., 53, 226.

Gaillard C., 1972 . Communication personnelle.

Höfliger H., I952 a. Über Drüsenaplasie in Eutervierteln des Rindes. Schweiz. Arch. Tierheilk., 94, $347-355$

HÖFliger H., 1952 b. Drüsenaplasie und -hypoplasie in Eutervierteln des Rindes, eine erblich bedingte Entwicklungsanomalie. Schweiz. Arch. Tierheilk., 94, 824-833.

KöNig H., r959. Zur Pathologie des Bullenshodens. Dtsch. Tierärztl. Wschr., 66, 65-7o.

KöNig H., I96r. Zur Pathologie der Geschlechtsorgane beim Stier. Habil. Schr. Bern et Arch exp. Vet. med., 16, 5or-584, I962.

König H., Weber W., Kupferschmied M., 1972. Zur Nebenhodenaplasie beim Stier und Eber. Schweiz. Arch. Tiezheilk., 114, 73-82.

KüNG W. B., r956. Weiterer Beitrag zur Kenntnis einer erblich bedingten Drüsen-Aplasie und Hypoplasie in Eutervierteln des Rindes. Vet. med. Dis. Zurich.

Lalouel J.-M., I970. Concentrations locales d'affections héréditaires rares. L'Expansion scientifique française, Paris.

Lauvergne J.-J., I967. Liste des travaux concernant les anomalies congénitales héréditaires ou nondes bovins dans le monde depuis 1900. Commission d'Étude de la F. E. Z., Session d'Oslo, I967. Commission de Génétique, ronéoté.

Lauvergne J.-J., r968. Catalogue des anomalies héréditaires des bovins (Bos taurus L.). Bull. tech. Dép. Génét. anim. (Inst. nat. Rech. agron., Fr.). No I, 9ז.

Lauvergne J.-J., Winzenried H. U., r969. Déterminisme héréditaire et extension de la ceinture blanche et des flancs colorés en race brune des Alpes de Suisse (note). Ann. Génét. Sél. anim., 1, I76.

Rizotti M., Aureli G., Zanotti L., Straini Pannelli R., Succi G., 1969. Ricerche morphologiche e istochimiche sulla pelle e biofisiche sui peli di bovini albini. Riv. Istoch. norm. pat., 15, 9 I-I Io.

Weber F., I972. Communication personnelle. 
Weber W., 1964. U̇ber Art, Häufigkeit und Genfrequenz der Missbildungen unserer Haustiere nebst einem Fall von Agenesis des Geruchapparates bei einem Kalb. Schweiz. Arch. Tierheilk., 88, $497-507$.

Weber W., Ig60. Blinde Kälber bei einer schweizerischen Rinderrasse. Schweiz. Arch. Tierheilk., 102, I 5-I8.

Weber W., I962. Dyschondroplasie bei Kälbern der Schwarzfleckviehrasse. Schweiz. Arch. Tierheilk., 104, 67-76.

Weber W., 1963. Erbliche Missbildung bei der Schwarzfleckviehrasse. Schweiz. Arch. Tierheilk., 105, 289-294.

Weber W., I963. Grundsätzliche Betrachtungen über die Pathogenetik. Schweiz. Arch. Tierheilk., 105, 672-678.

Weber W., r969. Albinokalb aus simmentaler Eltern. Schweiz Arch. Tierheilk., 111, 66I.

Weber W., Lauvergne J.-J., I964. Trois cas d'albinisme rencontrés en Suisse dans la race brune des Alpes. Ann. Zootech., 13, I5I-I54.

Weber W., Lauvergne J.-J., Winzenried H.U., I973. Albinisme héréditaire en race tachetée rouge de Suisse. Schweiz. Arch. Tierhielk., 115, (sous presse).

Winzenries H. U., 1972. Appels aux collégues actifs en pratiques bornie cécité congénitale chez le veau. Schweiz. Arch. Tierhielk. 114, 450.

Winzenried H. U., Lauvergne J.-J., I968. Albinism among brown swiss cattle. XII e Int. Conf. Genet., 1, 280 .

Winzenried H. U., Lauvergne J.-J., I97o. Spontanes Auftreten von Albinos in der schweizerischen Braunviehrasse. Schweiz. Arch. Tierheilk, 112, 581-587.

Zanotri L., Aureli G., Rizzotri M., Succi G., 1967. Osservazioni su peli di bovini albini mediante lo studio di spettri EPR. Riv. Istoch. Norm. Pat., 13, 451-46o. 